- Retraction note•

\title{
Erratum to: A simulation approach: Miscible carbon dioxide injection in a carbonate reservoir
}

(C) China University of Petroleum (Beijing) and Springer-Verlag Berlin Heidelberg 2010

Retraction: Ehsan Heidaryan, Jamshid Moghadasi and Marylena Garcia Quijada. A simulation approach: Miscible carbon dioxide injection in a carbonate reservoir. Petroleum Science 2010, 7(2): 257-262. DOI: 10.1007/s12182-010-0030-Z

This article is retracted because of copyright violation. 